Präv Gesundheitsf 2016 · 11:1-2 DOI 10.1007/s11553-015-0526-x

Online publiziert: 4. Januar 2016

c) Springer-Verlag Berlin Heidelberg 2015

\title{
CrossMark
}

Am 7. Juli 2015 ist Prof. Dr. med. Dr. med. dent. Wilhelm Kirch, langjähriger Direktor des Instituts für Klinische Pharmakologie der Medizinischen Fakultät der Technischen Universität Dresden und Sprecher des Forschungsverbundes Public Health Sachsen, verstorben.

Professor Kirch - 1947 in Köln geboren - studierte 1967-1974Zahnmedizin und Medizin in Mainz. Er promovierte 1975 im Fach Zahnheilkunde, 1976 im Fach Medizin. 1975-1982 war er an der I. Medizinischen Universitätsklinik in Mainz tätig und erhielt die Facharztanerkennung als Internist, Teilgebiet Nephrologie. 1982-1986 war er als Oberarzt an der Medizinischen Klinik und Poliklinik der Universität Essen tätig, wurde 1983 im Fach Innere Medizin habilitiert und 1985 auf eine C2-Professur für Allgemeine Innere Medizin berufen. 1986 wurde Professor Kirch Leitender Oberarzt an der I. Medizinischen Universitätsklinik in Kiel. Hier erhielt er 1988 die Fachanerkennung für Klinische Pharmakologie. 1993 wurde er auf den Lehrstuhl (C4) für Klinische Pharmakologie und Therapie am Institut für Klinische Pharmakologie der Medizinischen Fakultät der TU Dresden berufen. Im selben Jahr nahm Professor Kirch in Dresden auch die Ernennung zum Vorstandssprecher des BMBF-Forschungsverbundes Public Health Sachsen an. Er war dem Themengebiet Public Health eng verbunden und führte diesen Verbund mit nahezu 100 Projekten auf nationaler und internationaler Ebene sehr erfolgreich. 1994-1999 hatte er das Amt des Prodekans bzw. Dekans der Medizinischen Fakultät der TU Dresden inne. Professor

Joachim Fauler

Institut für Klinische Pharmakologie, Forschungsverbund Public Health Sachsen,

Med. Fakultät der TU Dresden, Dresden, Deutschland

\section{Nachruf für Prof. Dr. med. Dr. med. dent. Wilhelm Kirch (*04.07.1947-† 07.07.2015)}

Kirch war Mitglied in zahlreichen Fachkommissionen und Ausschüssen. 20002008 war Professor Kirch Vorsitzender des BMBF-Gutachtergremiums für die dritte Förderphase der ostdeutschen Medizinischen Fakultäten (NBL 3, Fördervolumen 96 Mio. €). Darüber hinaus war er in der Fachkommission Klinische Pharmakologie des Wissenschaftsrates der Bundesregierung und Mitglied im Sachverständigenausschuss beim Bundesinstitut für Arzneimittel und Medizinprodukte. Außerdem war er Mitglied der Arzneimittelkommission sowohl der Deutschen Ärzteschaft als auch Zahnärzteschaft. Des Weiteren begleitete Professor Kirch über viele Jahre das Amt des Vorsitzenden der Ethikkommission der TU Dresden, zu deren Ehrenvorsitzender er im Februar 2015 ernannt wurde.

Im Bereich der Klinischen Pharmakologie engagierte sich Professor Kirch in verschiedenen Fachgesellschaften. Er war 2009-2012 Präsident der Deutschen Gesellschaft für Experimentelle und Klinische Pharmakologie und Toxikologie e. V. Sein großes Engagement in diesem Bereich zeigen auch jene Kongresse, die auf diesem Gebiet unter seiner Federführung in Dresden sehr erfolgreich und mit hochrangigen Fachvertretern veranstaltet wurden:

- 7. Jahrestagung für Klinische

Pharmakologie, 03.-05.11.2005, Dresden,

- 78. Jahrestagung der DGPT, die vom 19.-22.03.2012, Dresden.

Im Laufe der Jahre verfasste Professor Kirch mehr als 650 Originalpublikationen. Zudem war er Verfasser und Heraus- geber von 53 Fachbüchern, u. a. von der „Encyclopedia of Public Health“ (Springer, New York, 2008), von „Public Health in Europe " (Springer, Heidelberg, 2004), der „Praktischen Arzneimitteltherapie“ (Springer, Heidelberg, 4. Aufl., 2006, mit J. Frölich), der „Arzneimitteltherapie“ (Thieme, Stuttgart, 4. Aufl., 2012, mit G.A. Kullak-Ublick), von „Aspekte der Prävention“ (Thieme, Stuttgart, 2009) und der "Prävention und Versorgung“ (Thieme, Stuttgart, 2012).

Professor Kirch fungierte als Chefherausgeber der Zeitschrift Prävention \& Gesundheitsförderung, die er $2005 \mathrm{zu}-$ sammen mit dem Springer-Verlag begründete. Gleichzeitig war er Editor in Chief der englischsprachigen Zeitschrift Journal of Public Health (Springer-Verlag), deren Wechsel er von der deutschsprachigen Zeitschrift für Gesundheitswissenschaften (Juventa-Verlag) erfolgreich forciert hatte. Zudem war er Rubrikenherausgeber in der Zeitschrift Medizinische Klinik (Springer-Verlag) sowie Deutsche Medizinische Wochenschrift (Thieme-Verlag). In all den Jahren war ihm die Zeitschriftenbetreuung eine Herzensangelegenheit.

Mit dem Wechsel nach Dresden etablierte er den Studiengang Public Health und den BMBF-Forschungsverbund Public Health an der Medizinischen Fakultät der TU Dresden. 1996-2005 war er Vizepräsident der Deutschen Gesellschaft für Public Health (DGPH) und wurde Vorstandsvorsitzender des Deutschen Verbandes für Gesundheitswissenschaften \& Public Health (DVGPH). 2002 erfolgte in Dresden unter Leitung von Professor Kirch die 
Ausrichtung des 10. Kongresses der „European Public Health Association“ (EUPHA) mit mehr als 1000 Teilnehmern. 2003 wurde Professor Kirch Präsident der „European Public Health Association“. In dieser Zeit gelang ihm auch die Beteiligung an hochdotierten EU-Projekten, u. a. koordinierte er die EU-Geschäftsstelle der "Coordinating Party“ für alle europäischen Projekte zum Thema „Lifestyle and Health Determinants" der Europäischen Gesundheitsbehörde in Luxemburg (DG-Sanco). Mit Tatkraft brachte er sich sehr erfolgreich in das Gebiet Public Health ein und veranstaltete hierzu eine Reihe von nationalen Kongressen:

- Nationaler Präventionskongress, 01.02.12.2005, Dresden,

- Nationaler Präventionskongress \& 6. Deutscher Kongress für Versorgungsforschung, 24.-27.10.2007, Dresden,

- Nationaler Präventionskongress, 27.28.11.2009, Dresden,

- Nationaler Präventionskongress \& 11. Deutscher Kongress für Versorgungsforschung, 27.-29.09.2012, Dresden.

Für sein Engagement auf dem Gebiet der Klinischen Pharmakologie erhielt Professor Kirch zahlreiche Preise, wie den „Fellow des American College of Clinical Pharmacology“, Ludolf-KrehlPreis der Südwestdeutschen Gesellschaft für Innere Medizin, den Homburg-Preis der Regensburger Akademie für Ärztliche Wissenschaften und MedvantisForschungspreis des Deutschen Ärztetages. Für die Ausrichtung des EUPHAKongresses in Dresden erhielt Professor Kirch den Dresden Congress Award 2004 sowie den Preis der „European Public Health Association“. 2006 verlieh ihm die AOK Sachsen den wissenschaftlichen Förderpreis.

Herr Professor Kirch hat die Medizinische Fakultät der TU Dresden in den Aufbaujahren nachhaltig geprägt. Er hat seine Tätigkeit als Direktor des Instituts für Klinische Pharmakologie und Sprecher des Forschungsverbundes Public Health Sachsen mit außerordentlichem persönlichen Engagement vertreten.

Seine Kollegen und Mitarbeiter des Instituts für Klinische Pharmakologie sowie Forschungsverbundes Public
Health Sachsen werden ihn als einen tatkräftigen und energiebetonten Menschen in guter Erinnerung behalten.

\section{Korrespondenzadresse}

\section{Prof. Dr. med. J. Fauler}

Institut für Klinische Pharmakologie, Forschungsverbund Public Health Sachsen, Med. Fakultät der TU Dresden Fetscherstraße 74, 01307 Dresden Joachim.Fauler@tu-dresden.de 